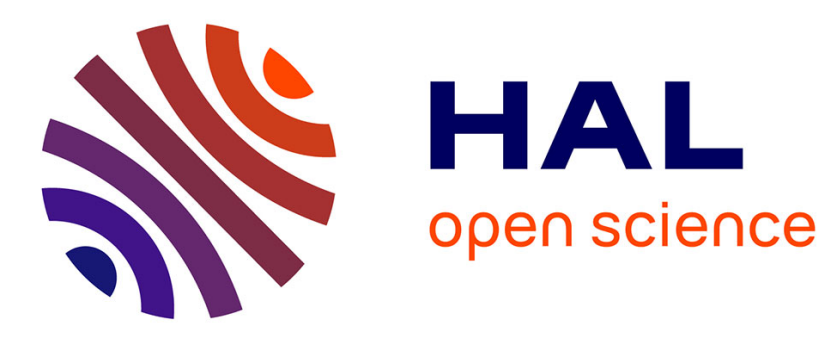

\title{
The physics of liquid water
}

\author{
J. Teixeira
}

\section{To cite this version:}

J. Teixeira. The physics of liquid water. Journal de Physique IV Proceedings, 1993, 03 (C1), pp.C1163-C1-169. 10.1051/jp4:1993114 . jpa-00251557

\section{HAL Id: jpa-00251557 https://hal.science/jpa-00251557}

Submitted on 1 Jan 1993

HAL is a multi-disciplinary open access archive for the deposit and dissemination of scientific research documents, whether they are published or not. The documents may come from teaching and research institutions in France or abroad, or from public or private research centers.
L'archive ouverte pluridisciplinaire HAL, est destinée au dépôt et à la diffusion de documents scientifiques de niveau recherche, publiés ou non, émanant des établissements d'enseignement et de recherche français ou étrangers, des laboratoires publics ou privés. 


\title{
The physics of liquid water
}

\author{
J. TEIXEIRA
}

Laboratoire Léon Brillouin, CEA-CNRS, CE-Saclay, 91191 Gif-sur-Yvette cedex, France

\begin{abstract}
Liquid water is a particularly complex molecular liquid. This is due essentially to the intermolecular hydrogen bond formation, the local tetrahedral symmetry and the short hydrogen bond lifetime. At low temperatures, the geometric aspects of the hydrogen bond network are dominant. A percolation approach of the spatial distribution of water molecules according to the number of their hydrogen bonds leads to a qualitative explanation of most of the thermodynamic anomalies observed at low temperature.

It is presented a short description of this percolation model and of its predictions of structural and dynamic properties of liquid water. Some selected experimental results are presented and intend to justify some of the hypothesis and main results of the theory.
\end{abstract}

The understanding of the fundamental mechanisms subtending the physics of liquid water at a microscopic level constitutes a tremendous challenge. The progress accomplished over the last years is partly due to the use of complex experimental techniques and to simulation procedures possible because of the rapid development of computer techniques.

At first sight, and in particular if one considers its simple chemical composition $\left(\mathrm{H}_{2} \mathrm{O}\right)$, the water molecule does not appear particularly complex; it could be a member of the family of molecules of the form $\mathrm{H}_{2} \mathrm{X}$ where $\mathrm{X}$ is an element of the sixth column of the periodic table of the elements, such as $\mathrm{H}_{2} \mathrm{~S}$. However, an extrapolation of the thermodynamic data of this class of materials to water shows that we are, in fact, in presence of a specific situation, due essentially to the formation of a large number of hydrogen bonds between molecules, as it was pointed out first by L. Pauling. Hydrogen bonds stabilize enormously the liquid structure of water; the melting and boiling temperatures, for example, are much higher than that of molecular substances of the same family.

A better understanding of the physics of water implies consequently a good knowledge of the physics of hydrogen bonds, which is not yet fully available.Moreover, there are other complications appearing when one studies liquid water at a molecular level. The geometrical shape of the molecule with its angle of $105^{\circ}$ between the two $\mathrm{OH}$ arms is at the origin of a very open tetrahedral coordination. Next-neighbour water molecules occupy the vertex of a tetrahedra around a central molecule and the hydrogen atoms are shared with disorder along the intermolecular lines. The resulting pentamer is an essential aspect of the water structure and it is found, with minor distortions for all the thermodynamic conditions of liquid water or, even, in the various forms of crystalline and amorphous ice. The recognition of the important role of the geometrical aspects imposed by hydrogen bonding is relatively recent and mainly due to the study of supercooled water, i.e., in metastable situations below $0^{\circ} \mathrm{C}$ at atmospheric pressure, when the peculiarities of water behaviour become evident.

Furthermore, the high dipole moment, dielectric constant and heat capacity make this liquid unique ; its role in earth sciences and biology is essential. That is why the understanding of the physics of water is not only a problem of the fundamental physics of liquids, but will have important consequences in more applied fields. 


\section{The water molecule}

Due to electronic hybridization of molecular orbitals, the water molecule has the shape of a V, with an angle (in liquid phase) of $105^{\circ}$ between $\mathrm{OH}$ arms. Two of the electron pairs of oxygen are non bonded and the other two electrons are shared with the two atoms of hydrogen. As a consequence, there is an excess of negative charge in one side of the molecule and an excess of the positive charge in the other side (the side of the hydrogen atoms). The resulting dipole moment is very high. It is then usual to represent the water molecule by a central core including the oxygen atom and four point charges distributed around it with tetrahedral symmetry. Two are positive and correspond to the hydrogen atoms and the two negative are the lone pairs. This picture is more or less assumed by several effective potentials extensively used to simulate the molecular dynamics of water in computer calculations. However, the real situation is less sharp ; actually, the charge distribution inside the water molecule is rather spherical, in particular on the lone-pair side.

When two water molecules are in presence they form very easily a bond - the hydrogen bond. The simple description that assumes point charges clearly shows that the hydrogen atoms will connect with the lone pairs of a neighboring molecule. The extrapolation of this geometrical picture to larger distances establishes an hydrogen bond network. This is actually the case, for instance, for the structure of common ice - the crystalline form $I_{h}$. It is characterized by a small coordination number : only four neighbours surround each molecule and there is "empty volumes" that favour hydrogen bond distortion and solubility properties.

It is worth noting that, in spite of its high association, i.e. the large extension of the hydrogen bond network, there is no possibility for long range order in liquid water, because of the presence of this large empty volume. More precisely, the structure of water at room temperature is characterized by a very stable pentamer with an almost invariable next-neighbours distance but, as well, by very broad distributions of the second and third neighbours distribution. The pair correlation function $g(r)$ shows clearly this feature; this function is directly related to the probability of finding an atom at distance $r$ of another taken as a reference at $\mathrm{r}=0$. This function, particularly important in liquids theory can be determined with high accuracy by $X$ ray or neutron scattering. The first technique is sensitive to the position of electronic clouds and, in the case of water, is dominated by the oxygen atoms. The measured $g(r)[1]$ is then essentially the oxygen-oxygen correlation function. Their peaks give the average position of oxygen atoms which almost coincide with the molecular centers; they are then easy to interpret.

It is interesting to compare the functions $\mathrm{g}(\mathrm{r})$ of liquid water and of an atomic liquid such as argon, the simplest reference for liquids theory. While for this last liquid oscillations in $g(r)$ are visible at large distances, for liquid water, the second peak is very broad and the position of the third is hardly identified. These curves translate the feature that the structural arrangement of argon atoms is very compact with a large coordination number (around 10), close to the highest compactness typical of Van der Waals substances. Instead, the water molecules never show, even at very low temperatures, an equivalent long range order. This is due, as we pointed out above, to the large free volume and in spite of very directed hydrogen bonds. More deeply, this feature is due to the very short life time of a bond, i.e., the time during which the energy of the hydrogen bond formed between two water molecules falls below some threshold, or, in geometrical terms, the time during which the shared hydrogen atom of the bond remain inside a narrow region around the alignment $\mathrm{O}-\mathrm{H}-\mathrm{O}$. This is why vicinal water, that is water molecules close to a subtract, having different properties of bulk water, is certainly reduced to a very small number of layers.

To summarize, the composition and geometry of the water molecule imply the three characteristics that, all together, make liquids water so specific : the large number of bonds, the tetrahedral symmetry and the short hydrogen bond life time. We will emphasize later these different aspects as well as some of the apparently contradictory consequences : large extent of the hydrogen bond connectivity, short distance molecular order and, as we will see later, a relatively large correlation length of the density fluctuations.

\section{Macroscopic aspects}

In liquids theory, the thermodynamic and transport properties of water are considered anormal. This is because the theory issued from Statistical Mechanics based on extrapolations of gas theory predicts temperature and pressure dependences of the liquids properties that are not observed in liquid water. As a typical example, consider the simple response function associated with density fluctuations; it is directly related to the isothermal compressibility. Almost intuitive arguments predict that such fluctuations increase with increasing temperature. One should expect, in consequence, an increase of compressibility with temperature. This is indeed observed for most liquids. However, liquid water below $46^{\circ} \mathrm{C}$ has the reverse behaviour : its isothermal compressibility increases faster when temperature is lower. Similar non conventional behaviour are observed with most of other properties. Some are particularly striking, such as the reduction of viscosity under pressure and have huge consequences in earth and life sciences. 
On the other hand, it is funny to think that water is almost the only natural liquid on the earth surface, that is present in all the physical states, and that it is the main constituent of living organisms. The sense of the concept of "water anomalies" is definitively not the common one ! It is interesting to remember that the second natural liquid present in the earth is silica and silicates. There are scarce data about these liquids, but at least some of their properties allow to classify them together with water. For instance, liquid silica shows a maximum on the temperature dependence of the density, like that observed for liquid water at $4^{\circ} \mathrm{C}$.

It has been tempting for long time to describe water properties, by combining the properties of an "ideal" liquid water having a classic behaviour and the properties of ice. This elegant way of describing water anomalies is at the origin of the success of several kinds of mixture or interstitial models. Very schematically, these models consider that liquid water is a mixture of two phases in strongly temperature dependent proportions. The argument is self-consistent, in the sense that the structure of ice represents like an ending point on the evolution of the structure of liquid water with decreasing temperature. In particular, ice is less dense than liquid water. These models are ruled out by small angle scattering experiments, but one may retain from them the fact that some density heterogeneities may play an important role.

Small angle scattering is the more appropriate technique to study this problem. It tests spatial heterogeneities on length scales slightly above the intermolecular distances. Such heterogeneities can have two origins. The first are the density fluctuations, always present in a liquid phase. As we have seen above they explain the compressibility of the liquid. Normally, the correlation length of density fluctuations of a liquid is negligible ; only close to the critical point, this length dramatically increases originating the well known phenomena of critical opalescence. The second origin of small angle scattering is due to the presence of a second component, such as a colloidal particle in the liquid. The scattering intensity is then related to the size of the particles. Also, a liquid mixture can show concentration fluctuations which appear very much like density fluctuations and increase also dramatically near demixtion. In practice, it is not always obvious to separate density from concentration fluctuations as both of them obey to the same Ornstein-Zernicke wave vector dependence. One way is to determine the zero-angle scattered intensity by careful extrapolation of the small angle data. The use of absolute units for the scattered intensity allows the comparison with thermodynamic data. In the case of pure density fluctuations, the zero-angle intensity, $S(O)$, is determined by the isothermal compressibility. In the case of concentration fluctuations, $S(O)$ depends on the osmotic compressibility. Experimental results show without ambiguity that the only density fluctuations are sufficient to explain the scattered intensity and this fact confirms that no ice-clusters are present in water. However a more careful analysis of the curves shows that the shape, of the scattered intensity, $S(Q)$, plotted versus the momentum exchange, $Q$, is not the usual one. The decreasing of $S(Q)$ with $Q$, particularly important at low temperatures, is unusual for a liquid far from its critical point. It indicates a correlation length of the density fluctuations that increases with decreasing temperature. With some arguments [2] it is possible to evaluate this correlation length to 2 or 3 intermolecular distances, a value that is, of course, very small, if compared with real critical behaviour, but that is significant and with an anomalous temperature dependence.

The presence of anomalous density fluctuations is consequently an experimental evidence. The fact that their intensity and spatial extension increase with decreasing temperature may suggest some kind of critical behaviour [3], in particular because the temperature dependence of thermodynamic properties have a qualitative behaviour analogous to the behaviour of a liquid when it approaches the spinodal line in the high temperature side of the phase diagram. Because the existence of a spinodal line on the solid-liquid side is unlikely and violates fundamental symmetry principles, it has been postulated [4] that it could correspond to a reentrant phase, i.e. that the spinodal line starting at the critical point could turn, at negative pressures around the triple point to appear again at atmospheric pressure around $-45^{\circ} \mathrm{C}$, i.e., close to the experimental nucleation temperature of hexagonal ice. This behaviour would be consistent with the existence of a temperature for which the liquid density passes a maximum. This interpretation does not solve the symmetry paradox consisting to predict when either a disordered gas phase or crystalline ice is formed after crossing of the spinodal line. Recent molecular dynamic computer simulations indicate the absence of loop in the spinodal line [5], and postulate the presence of a new metastable critical point, partly justified by the existence of two very different forms of amorphous ice.

The nature of the observed density fluctuations remains then a relatively open problem. Recent progresses are mainly due to simulations, due to the tremendous difficulties of experiments with water and amorphous ice under pressure and in metastable states. Whatever could be the definitive interpretation of the behaviour of water at low temperatures, in terms of thermodynamic quantities, it is also important to try to establish a picture at the molecular level.

\section{The percolation concept approach}

The fact that in liquid water there are density fluctuations with correlation lengths larger than the extension of structural order provides, probably, the central clue for understanding the water behaviour. Actually, the 
increase of the correlation length with decreasing temperature has to be associated in some way to the extension of the hydrogen bond network.

It is well known that the number of the so-called "intact" bonds is very high at all temperatures. This statement can be easily proved by Raman scattering experiments [6] or, even more easily noting the high latent heat of vaporization of water, due to the breaking of a large number of hydrogen bonds still present at boiling temperature.

In the usual language of percolation theories, one may suppose that water molecules occupy instantaneously sites of an irregular lattice with the local tetrahedric symmetry of crystalline ice. The formation or breaking of the bonds between neighbour molecules maps the bond percolation problem. Whatever is the evaluation of the number of intact bonds, this system will be above the percolation threshold. This means that, at a given moment, there are regions with some free molecules, but the largest majority of the water molecules are linked together forming an infinitely large cluster of hydrogen bonds. Of course, the lifetime of a given hydrogen bond configuration is not larger than the typical lifetime of a bond (of the order of $1 \mathrm{ps)}$. Otherwise the behaviour of water will be the behaviour of a gel. This instantaneous picture of water like a gel is clearly established by several experimental results and computer simulations.

Actually, the idea consisting to put the emphasis on geometrical aspects of the hydrogen bond network is originally due to Bernal and Fowler [7], who introduced the "continuum" models emphasizing the role of hydrogen bond distortion.

The use of percolation theories and the associated statistics allow the establishment of a pure geometrical interpretation of the behaviour of water at low temperatures [8]. The natural limitation of this approach is the absence of a potential. This is non conventional, but, at the present moment, all attempts to establish some ab initio treatment of the water problem failed completely. At low temperatures, such models are completely unable to describe the anomalies of water even qualitatively. Only efficient potentials that more or less impose the tetrahedral symmetry can reproduce the behaviour of water at low temperatures. Their use has been extremely useful and may be taken to test some predictions of the percolation theory. However, even now, there is no way for instance to justify the melting and boiling temperatures of water at atmospheric pressure ! The percolation model, in spite, or because of its intrinsic limitations is the only approach that can explain the anomalous behaviour of water at low temperatures.

This model has been presented and discussed several times. Consequently, I will simply summarize some of their main conclusions and hypothesis.

The first hypothesis assumes that the formation of bonds is completely random and only temperature dependent. This means, for instance, that, for a given molecule, the probability of formation of each of the possible four bonds is completely independent of the other bonds.

It is then easy to evaluate the populations of the five categories of molecules. They are given by the binomial distribution :

$$
f_{i}=\left(\begin{array}{c}
4 \\
i
\end{array}\right) p^{i}(1-p)^{4-i}
$$

where $f_{i}$ represents the population of molecules with $i$ bonds ( $i=0$ to 4 ) and $p$ the probability of forming a bond. Within the narrow temperature domain of liquid water, $p$ is almost a linear function of temperature :

$$
\mathrm{p}(\mathrm{T})=1.8-0.004 \mathrm{~T}
$$

because the energy of an hydrogen bond is much higher than the kinetic energy.

The central point of the percolation theory is that it builds a correlated distribution of the molecules, in spite of the randomness of the hydrogen bond formation. To understand this mathematical feature, it can be noted, for instance, that, if a molecule forms 4 bonds with their neighbours, these neighbours have at least one bond, or, in other words, it is clearly impossible to have a molecule belonging to the family $f_{0}$ next to another belonging to the family $f_{4}$. A careful study of the spatial distribution of the molecules of each family shows that there is a tendency for molecules with a high number of hydrogen bonds to group together in an almost segregative process. One may more or less identify the presence of clusters of, say, $\mathrm{f}_{4}$ molecules. This picture may look very much like a mixture model. However, we emphasize that instead of clusters, it is more exact to speak about regions with a high density of hydrogen bonds, or, even more exactly, that there is a correlation length for the distribution of highly bonded molecules that increases with $p$, then with decreasing temperature.

The next hypothesis of the percolation model assumes that molecules with a large number of bonds occupy a large volume than those with a small number of bonds. In other words, one may associate a molecular volume related to the family $f_{i}$ to which the molecule belongs. The volume increases with $i$. This strong hypothesis is confirmed by molecular simulations and X-ray experiments. The important consequence is the presence of density fluctuations with a non-zero correlation length. In this way, it is possible to justify the anomalous increase of compressibility with decreasing temperature. At low 
temperatures, the number of bonds increases and the density fluctuations increase as well, superimposed to the normally thermally driven density fluctuations. The combination of the two competing behaviours yields the compressibility minimum of the temperature dependence of isothermal compressibility.

The same argument can explain the increase of heat capacity with decreasing temperature observed at very low temperatures. Actually, heat capacity is related to entropy fluctuations. Regions of high density of bonded molecules are certainly more ordered, or have less distorted bonds, or have lower entropy than regions where dominates the presence of free or one-bond molecules.

The maximum of the density at $4^{\circ} \mathrm{C}$ is a consequence of the fact that the regions of molecules with a large number of bonds, correspond to both large molecular volumes and low entropy, an unusual situation due essentially to the high directionality of the hydrogen bonds and to the tetrahedral structure.

The presence of a second component - impurity, ion or dissolved material - breaks locally the hydrogen bond network. One can then understand why relatively small amounts of another substance (except heavy water) added to liquid water at low temperature, normally reduce or even suppress the water thermodynamic anomalies. This is true even for substances forming hydrogen bonds with water, such as alcohols. For instance, several percent of ethanol reduce the compressibility of water at $-20^{\circ} \mathrm{C}$ of a factor 2 [9].

The effect of pressure is more subtle. It does not change substantially the number of bonds, but the 0-00 angles are modified and the tetrahedral symmetry is partly lost. If one assumes that the probability function $\mathrm{p}(\mathrm{T})$, instead to count the number of intact bonds, measures more exactly the probability of forming a bond with the right tetrahedral symmetry, then it will be a decreasing function of pressure. Pressure, indeed, reduces the water anomalies.

\section{Dynamics}

The dynamics of water can also be understood in terms of the hydrogen bond network. One assumes first that the lifetime of the hydrogen bond has a small Arrhenius like temperature dependence. The problem after consists to establish the mobility of a labelled water molecule. If each hydrogen bond is formed with probability $\mathrm{p}(\mathrm{T})$ and has a characteristic life time $\tau_{\mathrm{HB}}$, then the possibility for a molecule to belong to the class $\mathrm{f}_{\mathrm{i}}$ during an arbitrary time interval $\tau_{\mathrm{HB}}$ is simply given by $\mathrm{f}_{\mathrm{i}}$.

For the purpose of dynamics, one must consider that the more efficient processes for water diffusion are rotational jumps around an hydrogen bond. More precisely, when a water molecule moves, most of the time it keeps one bond fixed and forms another bond with a neighbour molecule. In this picture, only molecules belonging to classes $f_{0}$ or $f_{1}$ are mobile ; the fraction $F_{I}$ of immobile molecules is given by :

$$
F_{I}(p)=f_{2}+f_{3}+f_{4}=6 p^{2}-8 p^{3}+3 p^{4}
$$

that is, a well -defined function of the temperature. In order to determine the residence time of an average molecule, we evaluate the time, $\tau_{0}$, during which a given molecule belongs to the ensemble $F_{I}$. The probability for a molecule to belong a time $\tau_{0}=n \tau_{\mathrm{HB}}$, to the ensemble $F_{I}$ is given by $F_{I}^{n}$. Consequently, the resident time is given by :

$$
\tau_{\mathrm{o}}=\tau_{\mathrm{HB}} \frac{\mathrm{cte}}{\ln \mathrm{F}_{\mathrm{I}}(\mathrm{p})}
$$

where the constant is of the order of unity.

With decreasing temperature, $p$ increases in an almost linear way; $F_{I}(p)$ has a stronger temperature dependence and $\tau_{0}$ diverges when $\mathrm{p}$ approaches 1 . This treatment shows that, even with a very weak temperature dependence of the hydrogen bond life time, $\tau_{\mathrm{HB}}$, the residence time has a dramatic temperature dependence, due to movement arrest resulting of the hydrogen bond formation. The simple equation (4) is in fair agreement with experimental data for dielectric relaxation time, self-diffusion and viscosity. It is interesting to remark that, in spite of its complexity and of the anomalous and strongly non-Arrhenius behaviour of all the transport properties of water, they respect approximatively the simplest relations, such as $\mathrm{D} \eta / \tau=$ constant [10], where $\mathrm{D}, \eta$ and $\tau$ stand for self-diffusion, viscosity and dielectric relaxation time, respectively. This is because all the transport properties are determined by the same mechanism. 


\section{Experimental aspects}

The ideas expressed above can be tested in detail by comparison with molecular dynamics computer simulation data. The agreement is always good and confirms the validity of the main hypothesis of the model. However, the main tests can be achieved only by performing comparisons with results issued from real experiments. As we pointed out above, the interesting region to study water anomalies is the low temperature region. At high temperatures, say above room temperature, classical thermal effects become more and more important masking the specific effects due to the hydrogen bond network. Consequently, it is important to obtain results at the lowest possible temperatures. This fact has been recognized relatively recently and mainly because of the fundamental paper published by Speedy and Angell [3].

Unfortunately, few experiments can really test the above model. Certainly, at the present state of our knowledge of the physics of water, all the experimental results are, at least compatible with the percolation model. However, some of these experiments are particularly close to the preceding picture. In this last paragraph we summarize some of their results.

Quasi-elastic neutron scattering is a technique that can probe the individual motions of hydrogen atoms, then, of water molecules. The sensitivity to individual motions is due to the incoherent scattering of neutrons by protons. Experiments performed at low temperatures and in supercooled regime [11] give an evaluation of the life time of an hydrogen bond, the molecular self-diffusion and the residence time. The two last quantities have a strongly non-Arrhenius temperature dependence, while the first is Arrhenius-like with an associated activation energy equal to the energy of the hydrogen bond. This experiment has moreover the merit to establish the mechanism of the intermolecular bond formation. Actually, it appears that librational movements are the more efficient for the breaking of the bonds. This statement is partly justified by the fact that the short hydrogen bond life time appears, technically speaking, like an hindered rotation movement. The Debye-Waller factor gives an evaluation of the critical angle between the actual position of an hydrogen atom when the bond breaks and the $\mathrm{O}-\mathrm{O}$ alignment - it is of the order of $30^{\circ}$. This indicates the order of magnitude of the amplitude of libration movements that are necessary to break a bond. The strong isotopic effect specific of water is explained by the fact that the higher mass of the deuteron makes the hydrogen bond more stable. As a consequence, the low temperature properties of liquid $\mathrm{D}_{2} \mathrm{O}$ are typically shifted 4 or $5^{\circ}$ above those of $\mathrm{H}_{2} \mathrm{O}$. The experiment gives also an evaluation of the jump length during the diffusion process ; it is of the order of $1.6 \AA$, i.e. of the $\mathrm{H}$-H distance, compatible with the proposed mechanism for diffusion by rotational jumps.

Another important experiment measures the propagation of collective excitations of very high frequency in liquid water [12]. In a classic liquid, collective excitations are sound waves normally studied either by ultra sounds or Brillouin light scattering techniques, probing pressure fluctuations. For non viscous liquids, one can observe only longitudinal waves ; their typical velocity is around $1500 \mathrm{~ms}^{-1}$, but the absorption strongly increases with increasing frequency. In most cases a weak dispersion is observed for frequencies of the order of the inverse of the structural relaxation time. Due to the typical values of wavelength of visible light, the maximum sound frequency that can be measured by Brillouin light scattering is of the order of some GHz. At this frequency, the sound modes are, in general, strongly damped. However, for some materials with a strong attractive well on the intermolecular potential, the damping is less severe and propagation may be observed at frequencies typically 1000 times larger, that is of the order of some THz. This is the case of some liquid metals such as rubidium or lead.

Water is certainly not a good candidate for such a observation. However, several molecular dynamics computer simulations [13] indicate that a second sound branch can exist at high frequencies. Its characteristic velocity is much larger than that of the normal sound and different from usual values observed with other molecular liquids.

Such high frequency collective modes can be probed by coherent inelastic neutron scattering, using triple-axis spectrometers, mostly used in studies of lattice dynamics of monocrystals. In spite of large error bars, this fast collective excitations are clearly identified propagating at velocities of the order of $3000 \mathrm{~ms}^{-1}$. It is true that several interpretations of this experimental result are possible. In particular, it could be the high frequency part of a very anomalous sound dispersion. It is also possible to interpret it as a collective mode propagating in the hydrogen bond network like in a gel. Because the network is forming and breaking with characteristic times of the order of a picosecond, these collective modes cannot be observed at low frequencies. Another way to express this consists to say that the collective excitation wavelength is of the order of the correlation length measured by small angle $\mathrm{X}$-ray scattering, that is some intermolecular distances.

The same collective excitations have been observed in situations where water is attached to a protein forming like a glove around it [14]. It is possible that, here too, one observes the propagation of a fast mode through the hydrogen bond network. 
Unfortunately, the difficulty and low accuracy of this kind of experiments and that the fact the region between light and neutron scattering is not experimentally accessible, avoids to use these results with more refinement.

\section{Conclusion}

In the preceding text, I emphasized the role of hydrogen bonds in liquid water. It is now clear that, at low temperatures, they play the major role. It is striking that some simple concepts issued from percolation are sufficient to explain some of the major anomalies of liquid water. However, other fundamental aspects, such as those related to chemistry, solvent properties and electric properties will imply a deep view of the water molecule. Also, it is important to remark that the properties of water in confined geometries are very different of those of bulk water. In some cases, water in confined geometries at room temperature has a behaviour similar to the behaviour of supercooled bulk water, in particular for the transport properties. Because, in many interesting situations, water is indeed confined in micropores, like in gels, or attached to a subtract like in porous silica or in biological macromolecules, the study of these situations is very important and actually is progressing rapidly. It is because of the leak of knowledge of the water behaviour that its role has been, for long time, either ignored or minimized. In the forthcoming years one can predict that the importance of the knowledge of water will be more recognized and that its study will progress rapidly.

\section{References}

[1] L. Bosio, S.-H. Chen and J. Teixeira, Phys. Rev. A, 27 (1983) 1468.

[2] L. Bosio, J. Teixeira, and H. E. Stanley, Phys. Rev. Lett., 46 (1981) 597.

[3] R.J. Speedy and C.A. Angell, J. Chem. Phys., 65 (1976) 851.

[4] R.J. Speedy, J. Phys. Chem., 86 (1982) 982, 3002.

[5] P.H. Poole, F. Sciortino, U. Essmann and H.E. Stanley, Nature, 360 (1992) 324

[6] G. d'Arrigo, G. Maisano, F. Mallamace, P. Migliardo and F. Wanderlingh, J. Chem. Phys., 75 (1981) 4264.

[7] J.D. Bernal and R.H. Fowler, J. Chem. Phys., 1 (1933) 515.

[8] H. E. Stanley and J. Teixeira, J. Chem. Phys., 73 (1980) 3404.

[9] O. Conde, J. Teixeira and P. Papon, J. Chem. Phys., 76 (1982) 3747.

[10] H. Pruppacher, J. Chem. Phys., 56 (1972) 101.

[11] J. Teixeira, M.-C. Bellissent-Funel, S.-H. Chen and A.J. Dianoux, Phys. Rev., A 31 (1985) 1913.

[12] J. Teixeira, M.-C. Bellissent-Funel, S.-H. Chen and B. Dorner, Phys. Rev. Lett., 54 (1985) 2685.

[13] A. Rahman and F.H. Stillinger, Phys. Rev. , A 10 (1974) 368 ; R.W. Impey, P.A. Madden and I.R. Mc Donald, Mol. Phys. , 46 (1982) 513.

[14] M.-C. Bellissent-Funel, J. Teixeira, S.-H. Chen, B. Dorner, H.D. Middendorf and H.L. Crespi, Biophys. J., 56 (1989) 713. 